\title{
CORRECTION
}

Malte Krack • Noha Aboulfotoh • Jens Twiefel • Jörg Wallaschek •

Lawrence A. Bergman • Alexander F. Vakakis

\section{Correction to: Toward understanding the self-adaptive dynamics of a harmonically forced beam with a sliding mass}

Published online: 19 October 2020

(c) Springer-Verlag GmbH Germany, part of Springer Nature 2020

\section{Correction to: \\ Arch Appl Mech (2017) 87:699-720 \\ https://doi.org/10.1007/s00419-016-1218-5}

The authors regret to have made an implementation error in their simulation code. More specifically, the harmonic base excitation had a wrong sign, which made it inconsistent with the contact kinematics. In the following, the figures affected by this error are presented in their original and corrected versions. The readers will see that the results are indeed very similar, but numerically not identical. There is only one exception which concerns the unsteady operation: The transient resonance capture encountered during the excitationlevel modulation was not observed with the corrected model for the given parameter set. Such a phenomenon may occur for a different parameter set. Besides this exception, all results remain qualitatively the same, and hence, the entire text of the original paper, including description, interpretation and conclusions, is in no way affected by the implementation error.

M. Krack $(\bowtie)$

Institute of Aircraft Propulsion Systems, University of Stuttgart, Pfaffenwaldring 6, 70569 Stuttgart, Germany

E-mail: malte.krack@ila.uni-stuttgart.de

N. Aboulfotoh $\cdot$ J. Twiefel $\cdot$ J. Wallaschek

Institute of Dynamics and Vibration Research, Leibniz Universität Hannover, Appelstr. 11, Hannover, Germany

L. A. Bergman

Department of Aerospace Engineering, University of Illinois at Urbana-Champaign, 104 S. Wright Street, Urbana, IL 61801, USA

A. F. Vakakis

Department of Mechanical Science and Engineering, University of Illinois at Urbana-Champaign, 1206 W. Green Street, Urbana, IL 61801, USA 

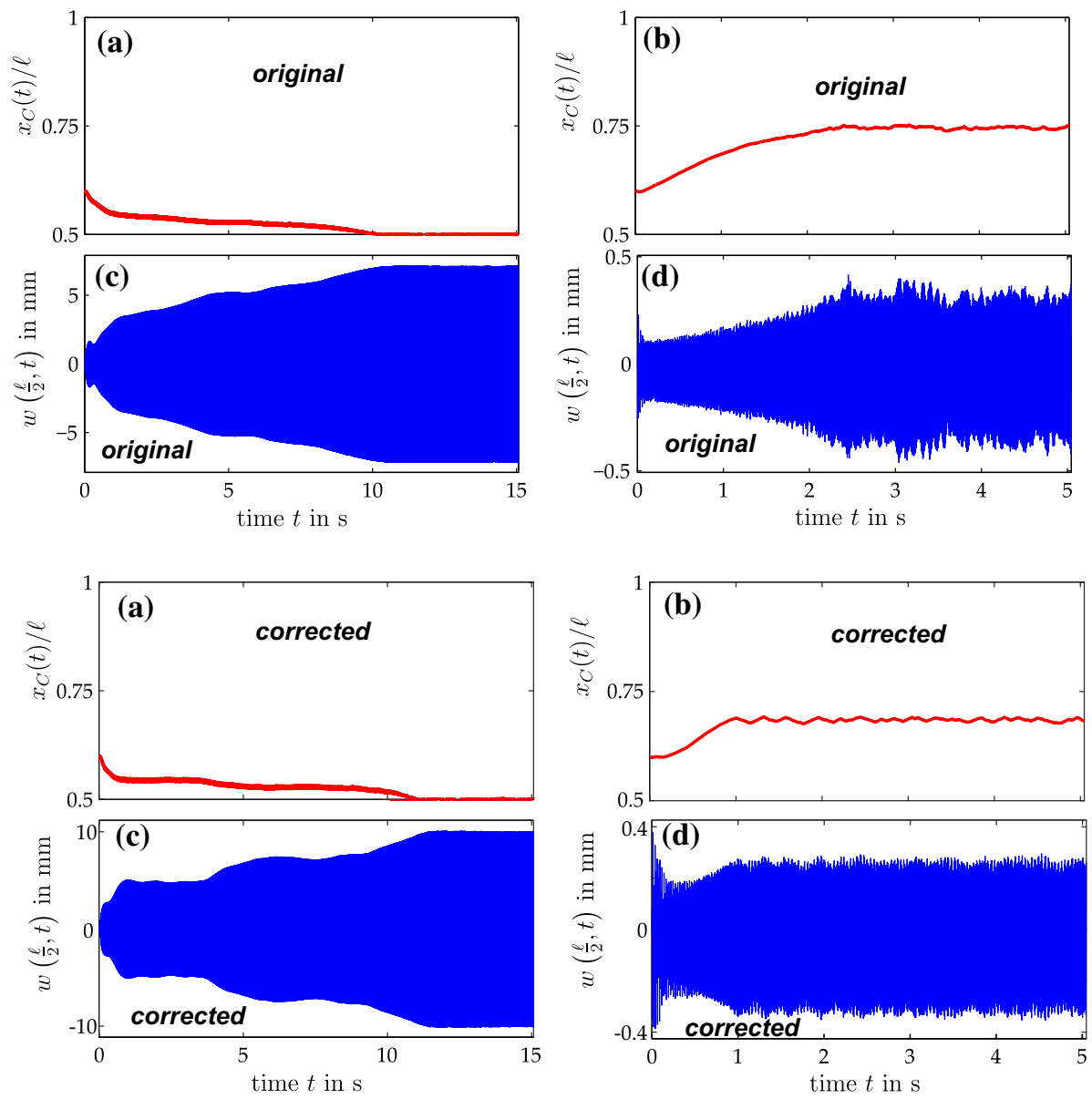

Fig. 3 Dynamical behavior for two different excitation frequencies: (a) and (c) $f_{\mathrm{ex}}=85 \mathrm{~Hz}$, (b) and (d) $f_{\mathrm{ex}}=125 \mathrm{~Hz}$; top: axial slider location, bottom: elastic displacement of the beam's center 

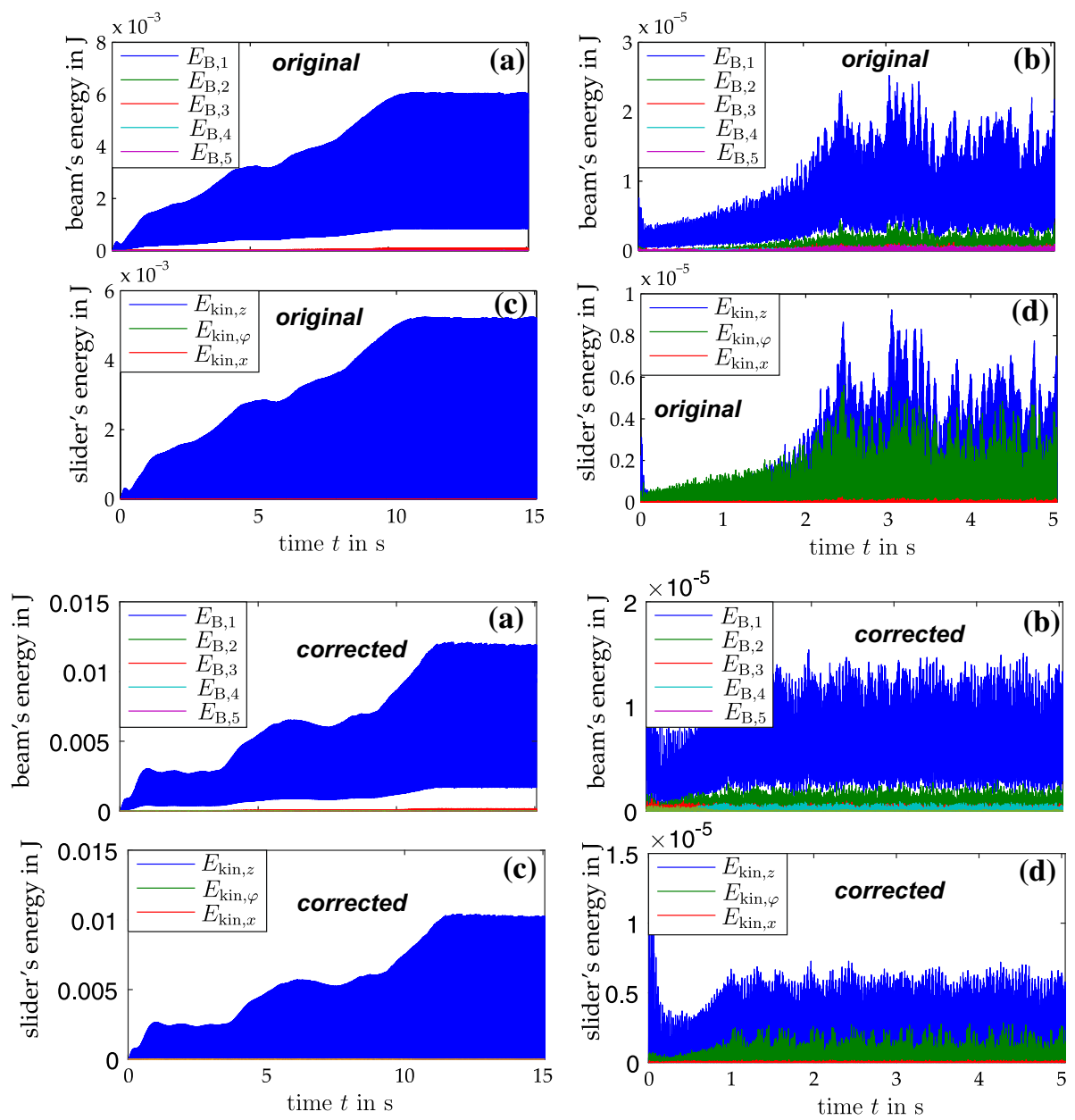

Fig. 4 Modal energy distribution for the two excitation frequencies considered in Fig. 4: (a) and (c) $f_{\mathrm{ex}}=85 \mathrm{~Hz},(\mathrm{~b})$ and (d) $f_{\mathrm{ex}}=125 \mathrm{~Hz}$; top: mechanical energy in the beam modes, bottom: kinetic energy of the slider 
original

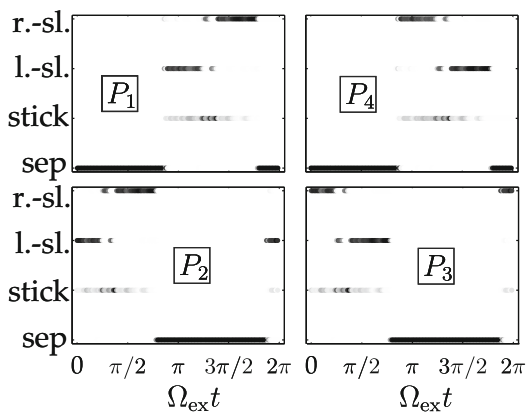

(a)

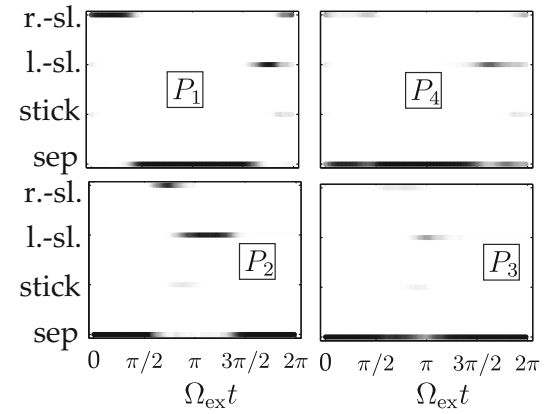

(b)

corrected
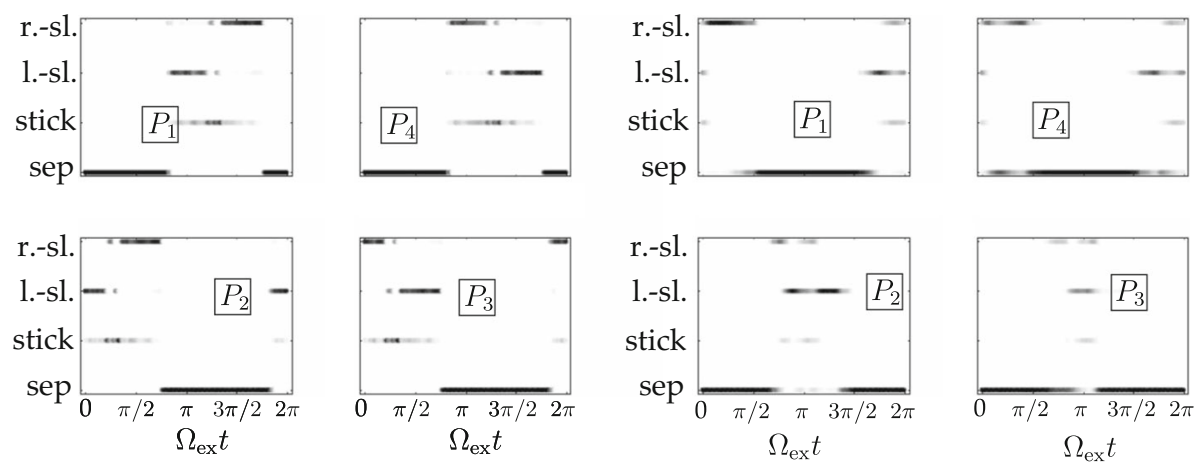

(a)

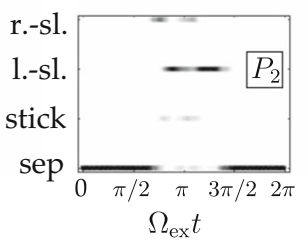

(b)

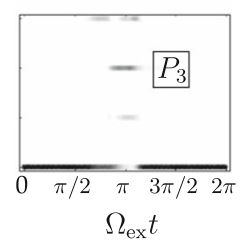

Fig. 5 Steady-state contact pattern for the two excitation frequencies considered in Fig. 4: (a) $f_{\mathrm{ex}}=85 \mathrm{~Hz}$, (b) $f_{\mathrm{ex}}=125 \mathrm{~Hz}$; the shading of the dots corresponds to occurrence over a sufficiently long steady-state time span

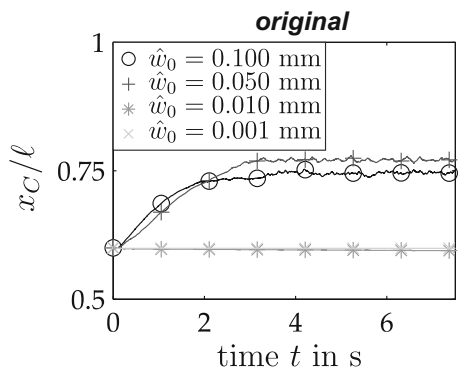

(a)

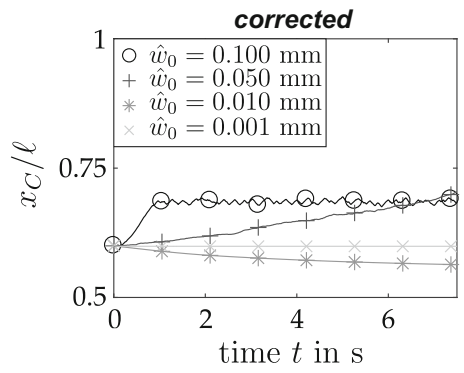

(a)

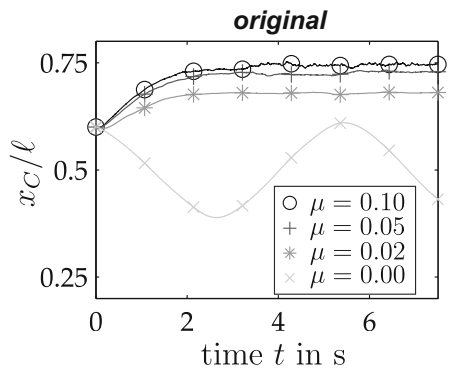

(b)

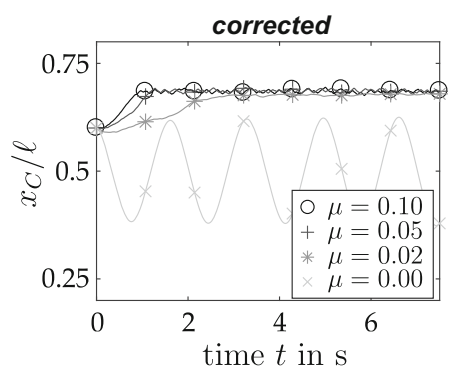

(b)

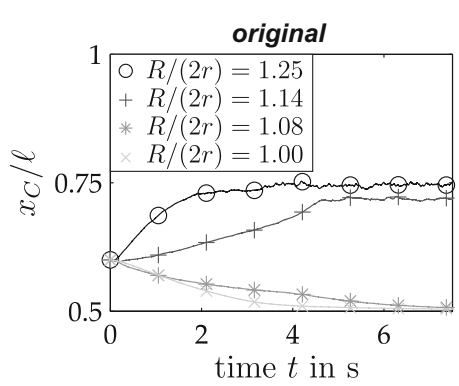

(c)

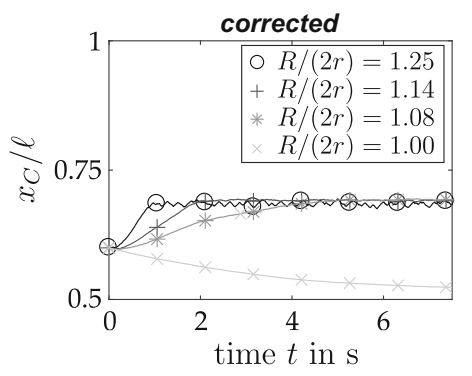

(c)

Fig. 6 Influence of certain parameters on the self-adaptivity for $f_{\mathrm{ex}}=125 \mathrm{~Hz}$ : (a) Effect of excitation level, (b) effect of friction, (c) effect of clearance 


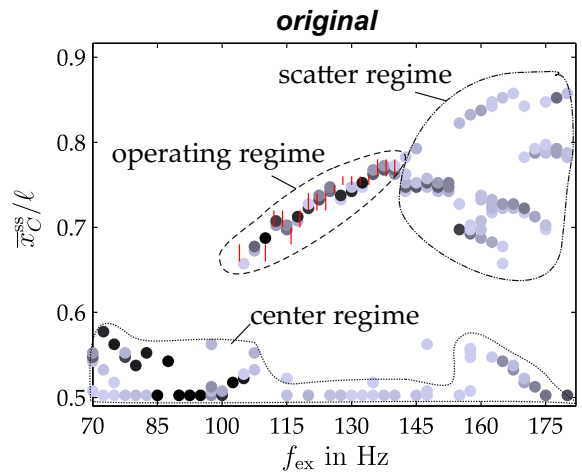

(a)

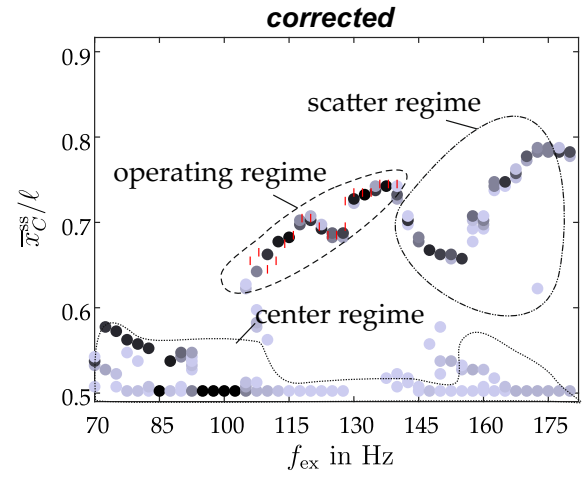

(a)

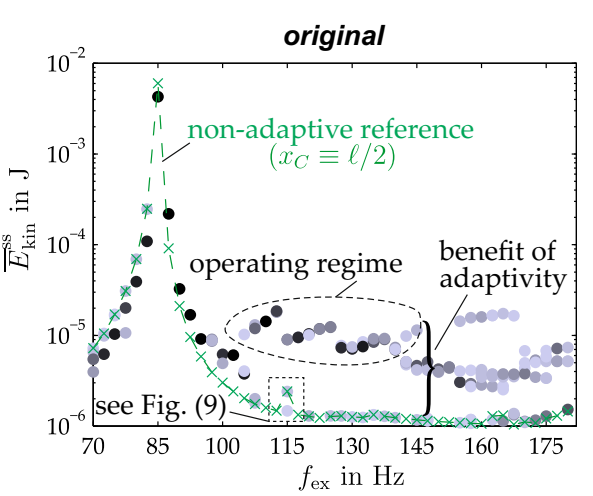

(b)

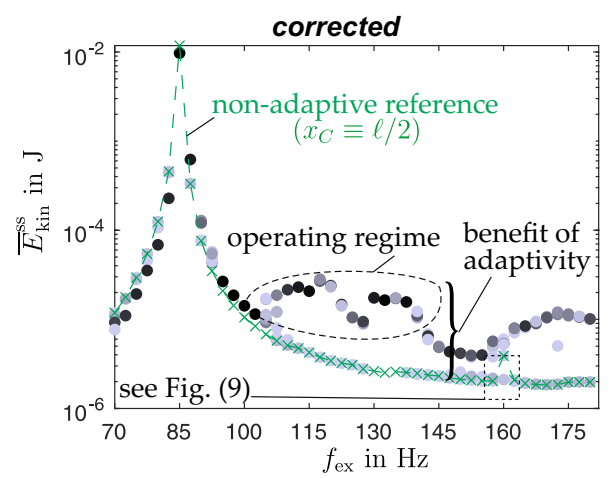

(b)

Fig. 7 Dependence of the steady-state behavior on the excitation frequency: (a) Axial slider location, (b) mechanical energy of the system; results were obtained for initial slider locations varied in the range $0.5 \ell \leq x_{C}(0) \leq \ell-b_{1} / 2$; the shading of the dots corresponds to their occurrence, as explained in the text 

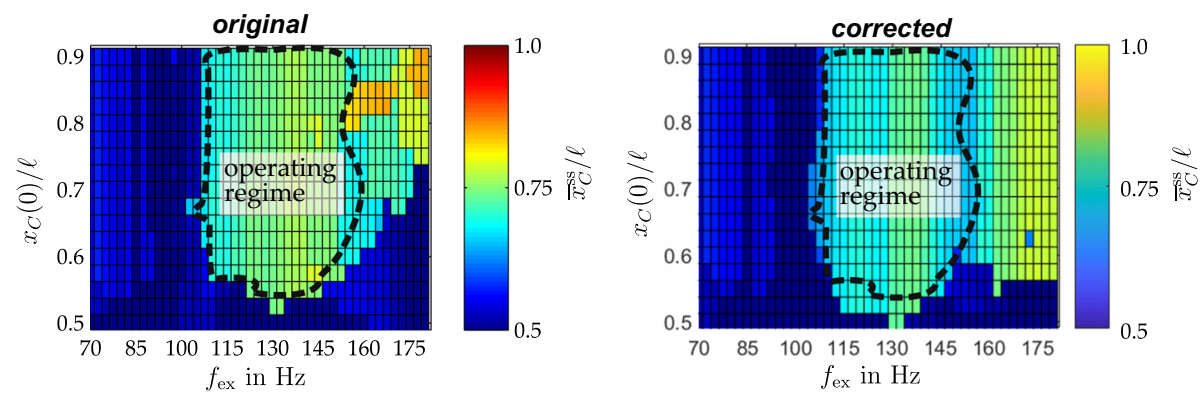

Fig. 8 Dependence of the steady-state axial slider location on its initial value $(t=0)$, as a function of the excitation frequency

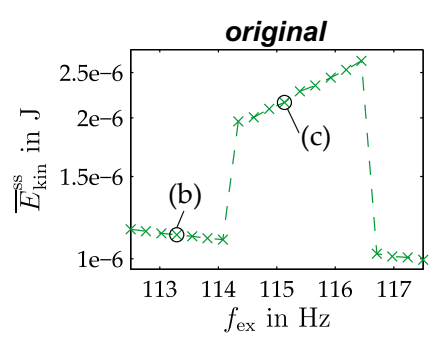

(a)

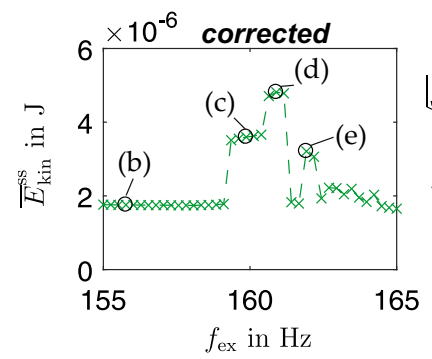

(a)

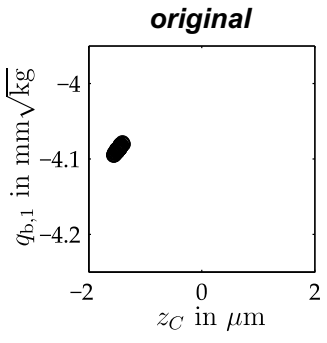

(b)

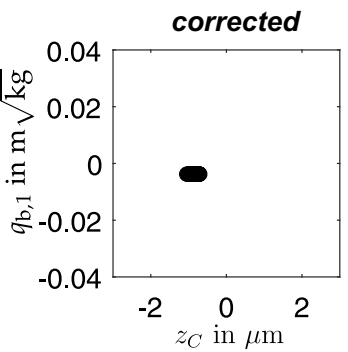

(b)

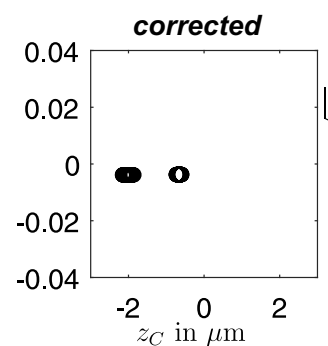

(d)

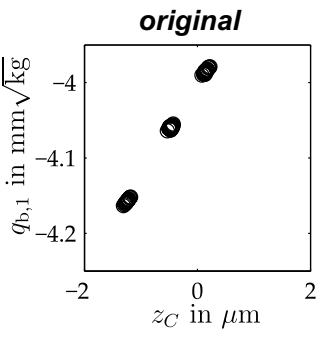

(c)

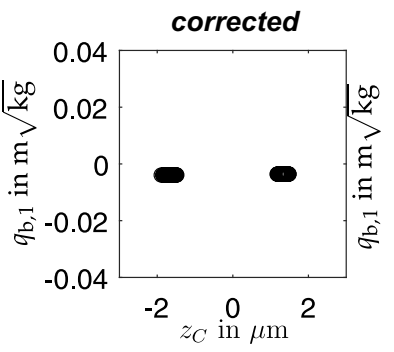

(c)

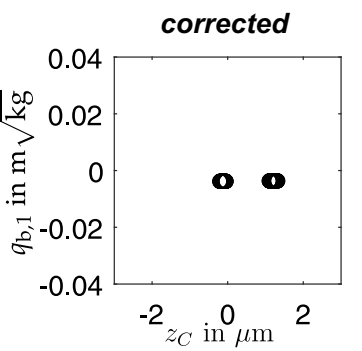

(e)

Fig. 9 A bifurcation of the non-adaptive reference system: (a) zoom into Fig. 7b, (b) to (e) Poincaré maps for the points indicated in (a) 


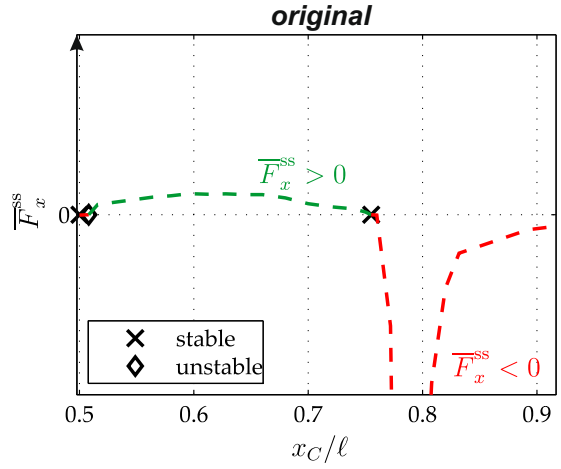

(a)

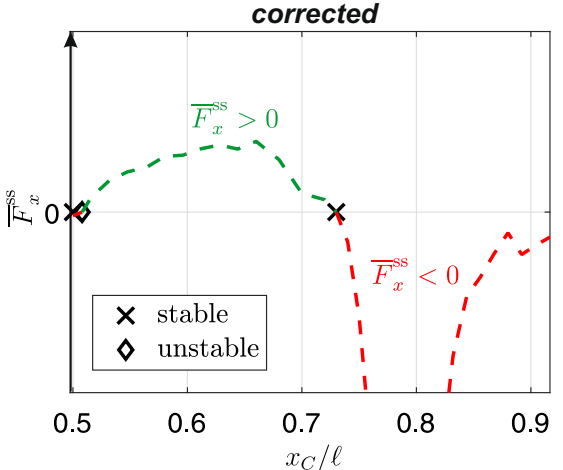

(a)

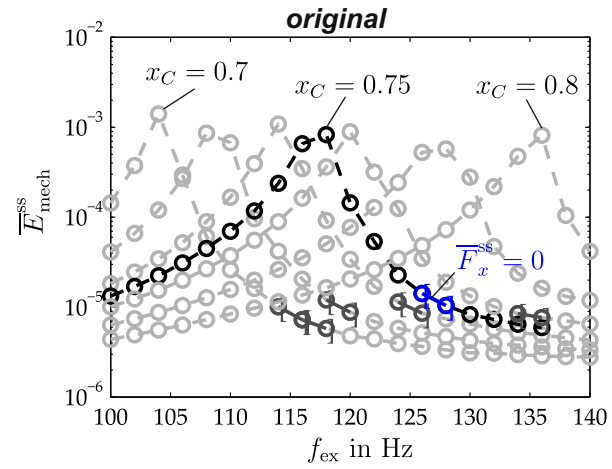

(b)

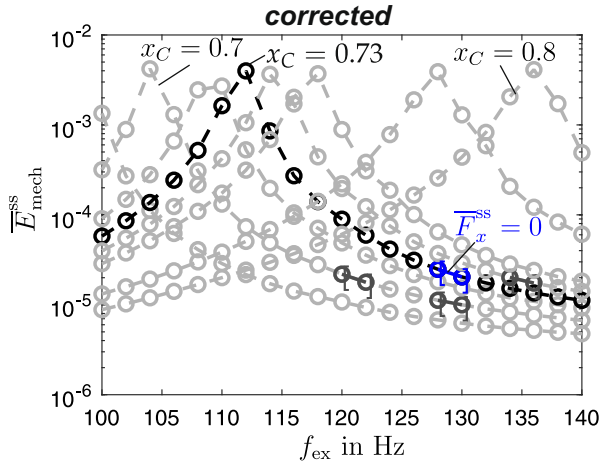

(b)

Fig. 11 Steady-state behavior of the surrogate system: (a) axial location dependence of the mean force and predicted fix points for $f_{\mathrm{ex}}=130 \mathrm{~Hz}$, (b) fixed-slider frequency response in terms of the mechanical energy

original

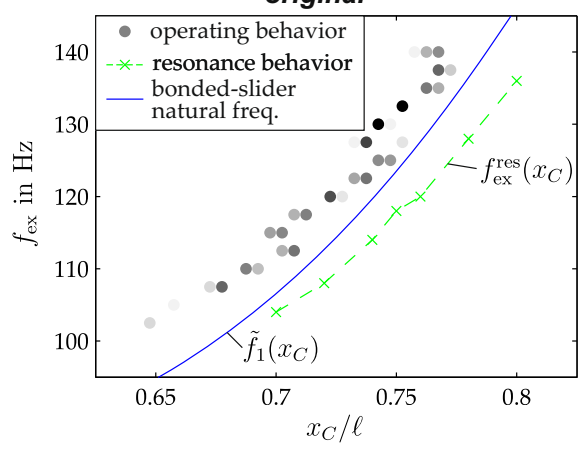

corrected

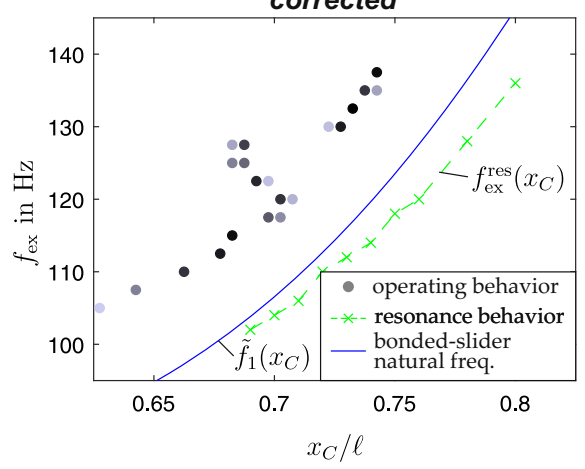

Fig. 12 Comparison of bonded-slider natural frequency, surrogate-based predicted resonance frequency and current operating behavior as a function of the slider coordinate 


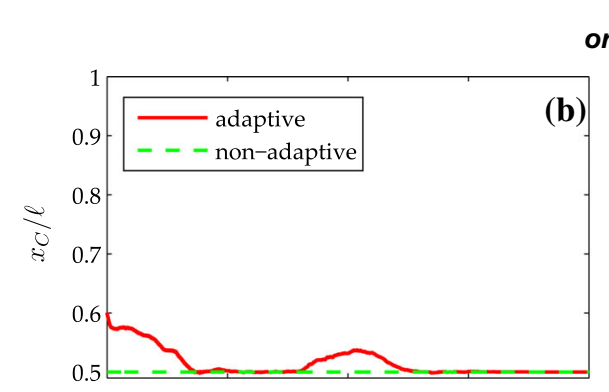

original
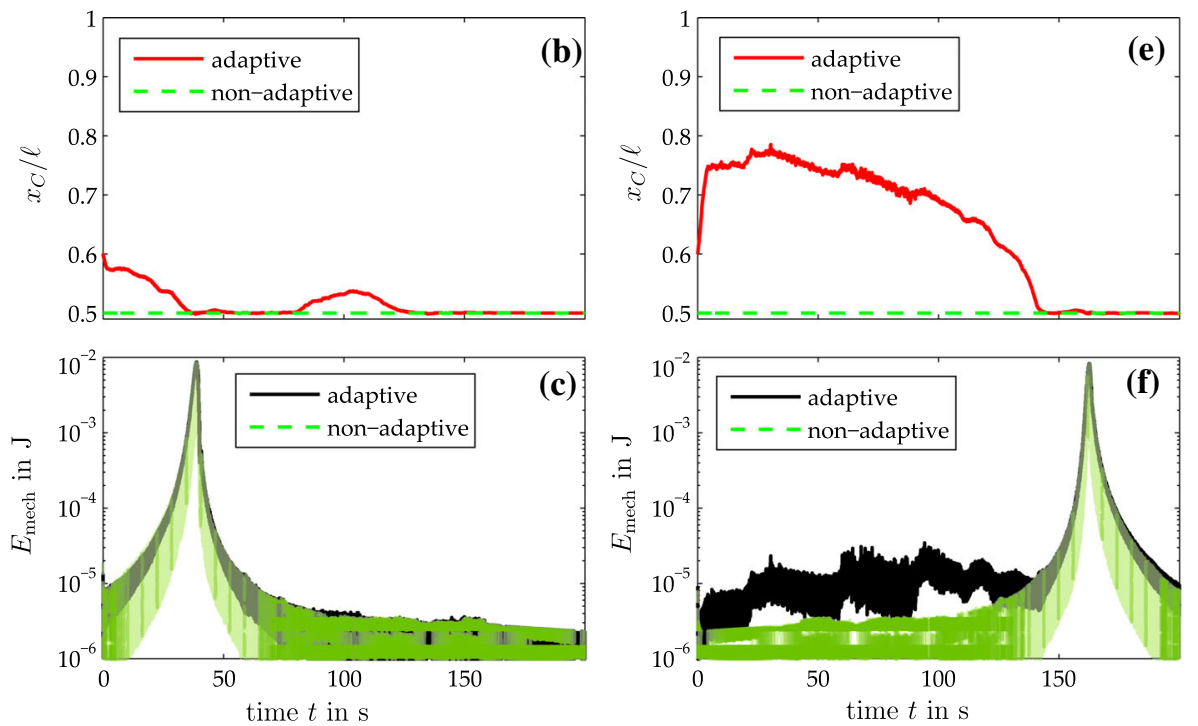

corrected
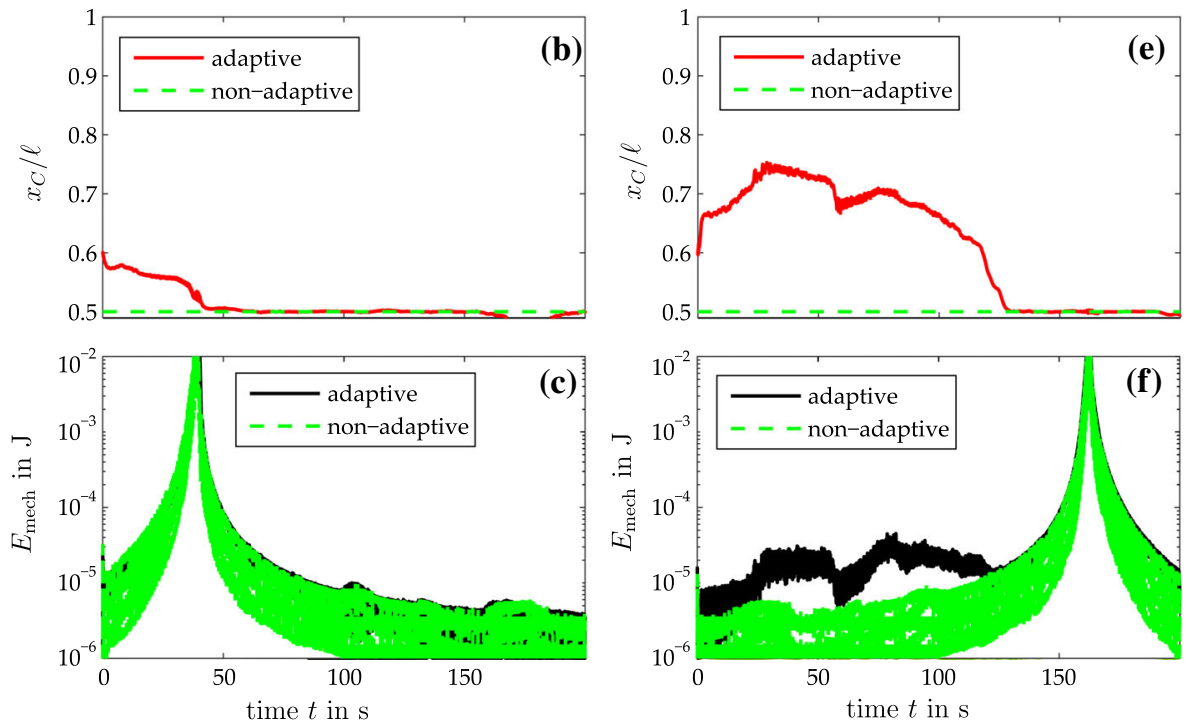

Fig. 13 Dynamical behavior for sine sweep excitation: (b)-(c) up-sweep, (e)-(f) down-sweep; top: axial slider location, bottom: mechanical energy

Publisher's Note Springer Nature remains neutral with regard to jurisdictional claims in published maps and institutional affiliations. 